\title{
Non-Invasive Detection of Mechanical Alternans Utilizing Photoplethysmography
}

\author{
Tudor Besleaga*, Sveeta Badiani, Guy Lloyd, Nicola Toschi, Senior Member, IEEE, Antonio \\ Canichella, Andreas Demosthenous, Fellow, IEEE, \\ Pier D Lambiase ${ }^{\dagger}$ and Michele Orini ${ }^{\dagger}$
}

${ }^{\dagger}$ Joint senior authors

\begin{abstract}
Background and Significance: Mechanical alternans (MA) is a biomarker associated with mortality and life-threatening arrhythmias in heart failure patients. Despite showing prognostic value, its use is limited by the requirement of measuring continuous blood pressure (BP), which is costly and impractical. Objective: To develop and test, for the first time, non-invasive MA surrogates based on photoplethysmography (PPG). Methods: Continuous BP and PPG were recorded during clinical procedures and tests in 35 patients. MA was induced either by ventricular pacing (Group $\mathrm{A}, \mathrm{N}=19$ ) or exercise (Group $\mathrm{B}, \mathrm{N}=16$ ). MA was categorized as sustained or intermittent if MA episodes were observed in at least 20 or between 12 to 20 consecutive beats, respectively. Eight features characterizing pulse morphology were derived from the PPG and MA surrogates were evaluated.

Results: Sustained alternans was observed in 9 patients $(47 \%)$ from Group A, whereas intermittent alternans was observed in 13 patients $(68 \%)$ from Group $A$ and in 10 patients $(63 \%)$ from Group B. The PPG-based MA surrogate showing the highest accuracy, $V^{\prime} M$, was based on the maximum of the first derivative of the PPG pulse. It detected both sustained and intermittent MA with $100 \%$ sensitivity and $100 \%$ specificity in Group $A$ and intermittent MA with $100 \%$ sensitivity and $83 \%$ specificity in Group B. Furthermore, the magnitudes of MA and its PPG-based surrogate were linearly correlated $\left(R^{2}=0.83, p<0.001\right)$. Conclusion: MA can be accurately identified non-invasively through PPG analysis. This may have important clinical implications for risk stratification and remote monitoring.
\end{abstract}

Index Terms - Blood pressure (BP), cardiac alternans, hemodynamic instabilities, mechanical alternans (MA), photoplethysmography (PPG),

\section{INTRODUCTION}

M ECHANICAL ALTERNANS (MA; aka blood pressure alternans and pulsus alternans) is a condition whereby blood pressure oscillates on an every other beat basis showing

Manuscript received June 22, 2018; revised October 05, 2018. This work was supported in part by University College of London (UCL) and Integrated Technologies Limited (www.itlmedical.com). Asterisk indicates corresponding author.

*T. Besleaga is with the Department of Medical Physics and Biomedical Engineering, UCL, London, U.K. (e-mail: tudor.besleaga.09@ucl.ac.uk).

S. Badiani and G. Lloyd are with Echocardiography Laboratory, Barts Heart Centre, St Bartholomew's Hospital, London, UK (e-mail: sveetabadiani@doctors.org.uk, Guy.Lloyd@ bartshealth.nhs.uk).

A. Canichella and N. Toschi are with Medical Physics, Department of Biomedicine and Prevention, University of Rome "Tor Vergata", Rome, Italy. an alternating sequence of strong-weak-strong-weak beats. The first observation of pulsus alternans was in a heart failure patient in 1872 [1]. Since then, there has been continuing interest in understanding the mechanisms and clinical manifestations of this phenomenon [2], [3].

Mechanical alternans is an established marker of cardiac impairment. Several studies have demonstrated an association between MA and heart failure [3]-[5], cardiomyopathy [6]-[8], ischemia [2], [9], aortic stenosis [10], [11] and life-threatening arrhythmias [2], [12]. The presence of MA has been sufficient to identify patients with cardiac risk in heart failure populations [3] and MA with sustained pattern with high magnitudes is linked to left ventricular disfunction [5].

Despite its clear potential as a risk marker, the use of MA is seriously limited by the requirement of continuous blood pressure monitoring which is done either invasively, for best accuracy, or performed through cumbersome and expensive non-invasive devices. In contrast to such continuous blood pressure (BP) monitoring, photoplethysmography (PPG) based devices e.g. pulse oximeters, are non-invasive, cheaper, convenient to use and widely available in hospitals and clinics. The current expansion of wearable devices allowing continuous PPG recordings [13] provides an opportunity for improving platforms for remote care monitoring.

The PPG technology measures hemodynamic changes by illuminating arterially perfused tissue with light in the visible and infrared spectrum. Arterial pulsations affect the intensity of light passing through the tissue, which is captured by a detector and forms the PPG waveform. Multiwavelength analysis permits the calculation of oxygenated blood in the local tissue, an application which today is rarely absent from patient monitors. Beyond its conventional use in oxygen saturation monitoring, the PPG technology has advanced in recent years

N. Toschi is also affiliated with A.A. Matinos Center for Biomedical Imaging, Massachusetts General Hospital and Harvard Medical School, Boston, USA. (toschi@med.uniroma2.it, canichella@med.uniroma2.it)

A. Demosthenous is with the Department of Electronic and Electrical Engineering, UCL, London, WC1E 7JE, U.K. (a.demosthenous@ucl.ac.uk).

M. Orini and P. D. Lambiase are with the Electrophysiology Department, Barts Heart Centre, St Bartholomew's Hospital, London, UK (e-mail: m.orini@ucl.ac.uk, p.lambiase@uc.ac.uk). P. D. Lambiase is also affiliated with the Institute of Cardiovascular Science, UCL, and acknowledges support from UCLH Biomedicine NIHR. M. Orini is also affiliated with the Department of Mechanical Engineering, UCL, and was supported by an IEF 2013 Marie Curie Fellowship. 
and is currently capable of detecting: pulse rate variability, as a surrogate of heart rate variability [14], respiratory rate[15], [16] sleep apnea [17]-[19], ectopic beats [20], heart rate turbulence [21] and atrial fibrillation [22], [23]. Estimation of blood pressure is another active area of research, however, achieving high accuracy remains challenging [24]-[26], particularly without patient-specific calibration. Rather than focusing on estimating BP, the aim of this study was to detect specific beat to beat blood pressure oscillations.

We hypothesized that PPG could also be used to detect MA, thus providing further insights into patient's health at low cost and new opportunities to improve cardiac risk stratification.

\section{METHODS}

\section{A. Data Sets and Preprocessing}

The study was approved by the local research ethics committee and all patients gave informed consent for data collection. In total, 39 patients were prospectively recruited. Four patients were excluded from the study due to the signal being corrupted by either nail polish on the subject's nail, or obstruction of the brachial artery during exercise. The 35 remaining patients were split in two analysis groups, as shown in Table I.

TABLE I

Data Collection Groups

\begin{tabular}{|c|c|c|c|}
\hline & \multicolumn{2}{|c|}{ Group A } & Group B \\
\hline$\underline{\text { Patients }}$ & $\underline{7}$ & $\underline{12}$ & $\underline{16}$ \\
\hline Setting & Cardiac Theatre & $\underline{\text { Catheter Lab }}$ & Outpatient Care \\
\hline Intervention & $\underline{\text { Cardiac Surgery }}$ & Catheter Ablation & $\underline{\text { CPET }}$ \\
\hline MA Induction & Ventricular Pacing & $\underline{\text { Ventricular }}$ & Exercise \\
\hline$\underline{\text { PPG }}$ & $\underline{\text { GE Carescape }}$ & $\underline{\text { GE Mac-Lab }}$ & iWorx PO-100U \\
\hline$\underline{\text { BP }}$ & $\frac{\text { GE Carescape }}{\text { Invasive }}$ & $\frac{\text { GE Mac-Lab }}{\underline{\text { Invasive }}}$ & $\frac{\text { FinometerPro }}{\text { Non-invasive }}$ \\
\hline
\end{tabular}

GE: General Electric; CPET: Cardiopulmonary exercise test.

Group A consisted of 19 patients: 12 patients undergoing catheter ablation of ventricular tachycardia and 7 patients undergoing open heart surgery for coronary artery bypass grafting. In the Catheterisation Lab invasive aortic blood pressure and PPG, from the finger, were simultaneously measured with sampling frequency equal to $240 \mathrm{~Hz}$, (Mac-Lab System, General Electric), whereas in the cardiac theatre invasive aortic blood pressure and PPG were simultaneously measured with sampling frequencies equal to $100 \mathrm{~Hz}$, (Carescape, General Electric). Ventricular pacing (cycle length equal to $505 \pm 59 \mathrm{~ms}$, range $410-870 \mathrm{~ms}$ ) was established to increase heart rate and induce MA, except in four patients who were studied while in incessant monomorphic stable ventricular tachycardia.

Group B consisted of 16 patients undergoing cardiopulmonary exercise testing (CPET). During the test, patients were positioned on a reclining cycle ergometer and exercised from mild to maximum effort. The test_followed a ramp protocol in which the pedaling workload was set to $0 \mathrm{~W}$ for the first minute and increased by 10 to $20 \mathrm{~W} /$ minute, depending on patient fitness. Exercise continued until exhaustion or reaching a target heart rate equal to $85 \%$ of the maximum predicted heart rate (calculated as 220-patient age), as per standard protocol. Non-invasive continuous peripheral blood pressure was recorded via a finger cuff with a FinometerPRO, Finapres Medical Systems. The raw finger pressure signal was exported via the device analog output and recorded via Matlab through a National Instruments USB-6002 at a sampling rate of $200 \mathrm{~Hz}$. The PPG was recorded from the finger with a PO2-100U sensor, iWorx, using an LabScribe v2.0; the sampling rate was $200 \mathrm{~Hz}$. Both PPG sensors used in Group A and B used infrared wavelengths to sense tissue volume variations. The peak wavelength was $940 \mathrm{~nm}$ for Group A and $910 \mathrm{~nm}$ for group B. Overall, the average recording time was $16 \mathrm{~m} 26 \mathrm{~s}( \pm 12 \mathrm{~m} 15 \mathrm{~s})$. For Group A it was $19 \mathrm{~m} 31 \mathrm{~s}( \pm 15 \mathrm{~m}$ $23 \mathrm{~s})$, and for Group B it was $12 \mathrm{~m} 47 \mathrm{~s}( \pm 5 \mathrm{~m} 28 \mathrm{~s})$.

A finite impulse response (FIR) low pass filter with a $30 \mathrm{~Hz}$ cut-off frequency was used to reduce noise in the PPG signals as well as in the BP signals recorded invasively through an arterial line (Group A). To reduce noise in the BP recordings provided by the non-invasive finger probe (Group B) a lower cut-off frequency of $10 \mathrm{~Hz}$ was required. The filter order was adjusted to the signal sampling frequency as half its value.

An automated peak selection algorithm was employed to detect each PPG pulse and its corresponding BP pulse. The algorithm evaluates the signal derivative, selects peak values over a threshold and excludes peaks which are less than $200 \mathrm{~ms}$ apart. Depending on the measurement location, PPG peaks follow BP peaks by an approximate time delay. We used a time window for this delay, to assess the accuracy of the PPG peaks allocation. Furthermore, to exclude bigeminy and ectopic induced oscillations from the MA analysis, beats accompanied by changes in cycle length of more than $200 \mathrm{~ms}$ were excluded.

\section{B. General Definition and Detection of Alternans}

Let us define a time series $\left\{X_{n}\right\}_{n=1}^{N}$ representing a sequence of values for a given pulse-specific feature extracted from either the BP or PPG signal; $n$ is the beat number and $N$ is the total number of beats. An alternating sequence of $Q$ beats was identified by an uninterrupted succession of beats, showing an alternating pattern (high-low-high-low), satisfying:

$$
\left\{\begin{array}{l}
\left(X_{n}>X_{n-1} A N D X_{n}>X_{n+1}\right) O R \\
\left(X_{n}<X_{n-1} A N D X_{n}<X_{n+1}\right)
\end{array}\right\}_{n=Q_{i}}^{Q_{f}}
$$

where $Q_{i}$ and $Q_{f}$ are the initial and final beat numbers of the alternating sequence, and $Q=Q_{f}-Q_{i}+1$.

Based on the value of $Q$, we categorized alternans episodes as Sustained, if the alternating pattern was uninterrupted for at least 20 consecutive beats $(Q \geq 20)$, and as Intermittent if the alternating pattern was uninterrupted for at least 12 , but no more than 19 , consecutive beats $(12 \leq Q<20)$.

Furthermore, the magnitude of the alternans episode was measured as:

$$
\overline{\Delta_{r} X}=\frac{1}{Q} \sum_{n=Q i}^{Q f} \frac{\left|\Delta X_{n}\right|}{\max \left(X_{n}, X_{n-1}\right)}
$$

where $\Delta X_{n}=X_{n}-X_{n-1}$, and $Q_{i}$ and $Q_{f}$ are the initial and final beat numbers of the alternans episode. 


\section{Definition of Mechanical Alternans}

As shown in Fig. 1, mechanical alternans was detected based on the dynamics of the maximum of the first derivative of the BP signal:

$$
X_{n}=P_{M}^{\prime}(n)=\max _{t \in I_{P I}}\left(\frac{d P(t)}{d t}\right)
$$

where $I_{P I}$ is the time interval within the $n^{\text {th }}$ heartbeat, and $t$ is time.

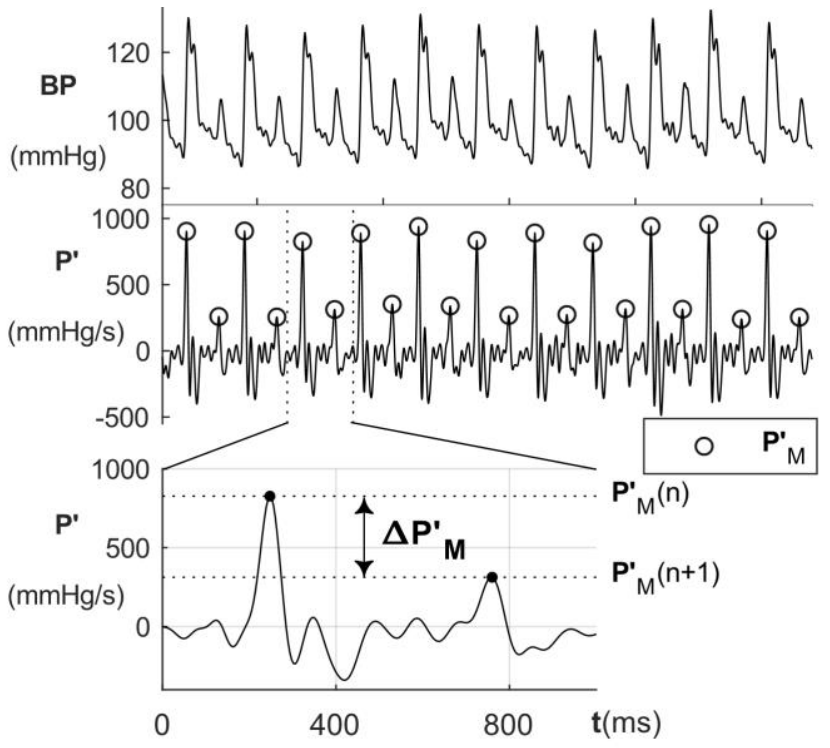

Fig. 1. Method of calculating alternans magnitude. Recording showcasing sustained alternans present in systolic BP, in the first derivative of BP, P', and the absolute magnitude calculated as the difference between two consecutive strong and weak peaks of $\mathrm{P}^{\prime}$.
A MA episode was classified as:

- Sustained MA (Fig. 2a) if the magnitude of the alternans episode was higher than $4 \%$ and the alternating pattern was uninterrupted for at least 20 consecutive beats $\left(\overline{\Delta_{r} P_{M}^{\prime}}>4 \%\right.$ and $\left.Q \geq 20\right)$;

- Intermittent MA (Fig. 2b) if the magnitude of the alternans episode was higher than $4 \%$ and the alternating pattern was uninterrupted for at least 12 , but no more than 19 , consecutive beats $\left(\overline{\Delta_{r} P_{M}^{\prime}}>4 \%\right.$ and $\left.12 \leq Q<20\right)$. Results obtained using different values for $Q$ and $\Delta_{r} P_{M}^{\prime}$ are presented in the supplementary Table S.I.

Patients that exhibited at least one episode of alternans were classified as sustained MA positive $\left(M A_{S}^{+}\right)$or intermittent MA positive $\left(M A_{I}^{+}\right)$, depending on the MA duration. Patients not showing MA were classified as MA negative $\left(M A^{-}\right)$.

\section{Feature Extraction and PPG-Based MA Surrogates}

Eight pulse-specific indices were defined and measured for each PPG pulse. Seven of these features are illustrated in Fig. 3 for a single PPG pulse, the $8^{\text {th }}$ being the pulse mean over the pulse interval. Beat-to-beat oscillations in these eight indices were then analyzed as previously described in section B. Sustained and intermittent MA was detected based on the number of consecutive beats showing an uninterrupted alternating sequence and the alternans magnitude $\overline{\Delta_{r} X}>\Delta X_{0}$, where $\Delta X_{0}$ is an optimal threshold maximizing accuracy detection estimated by receiver operating characteristic (ROC) analysis, which was performed by incrementally varying the threshold value from $0 \%$ to $60 \%$.

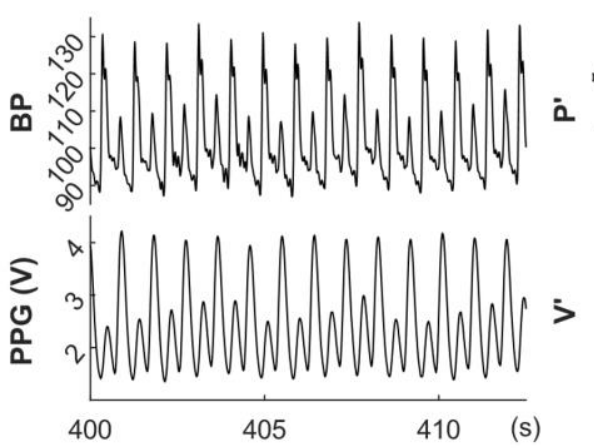

(a) Sustained Alternans
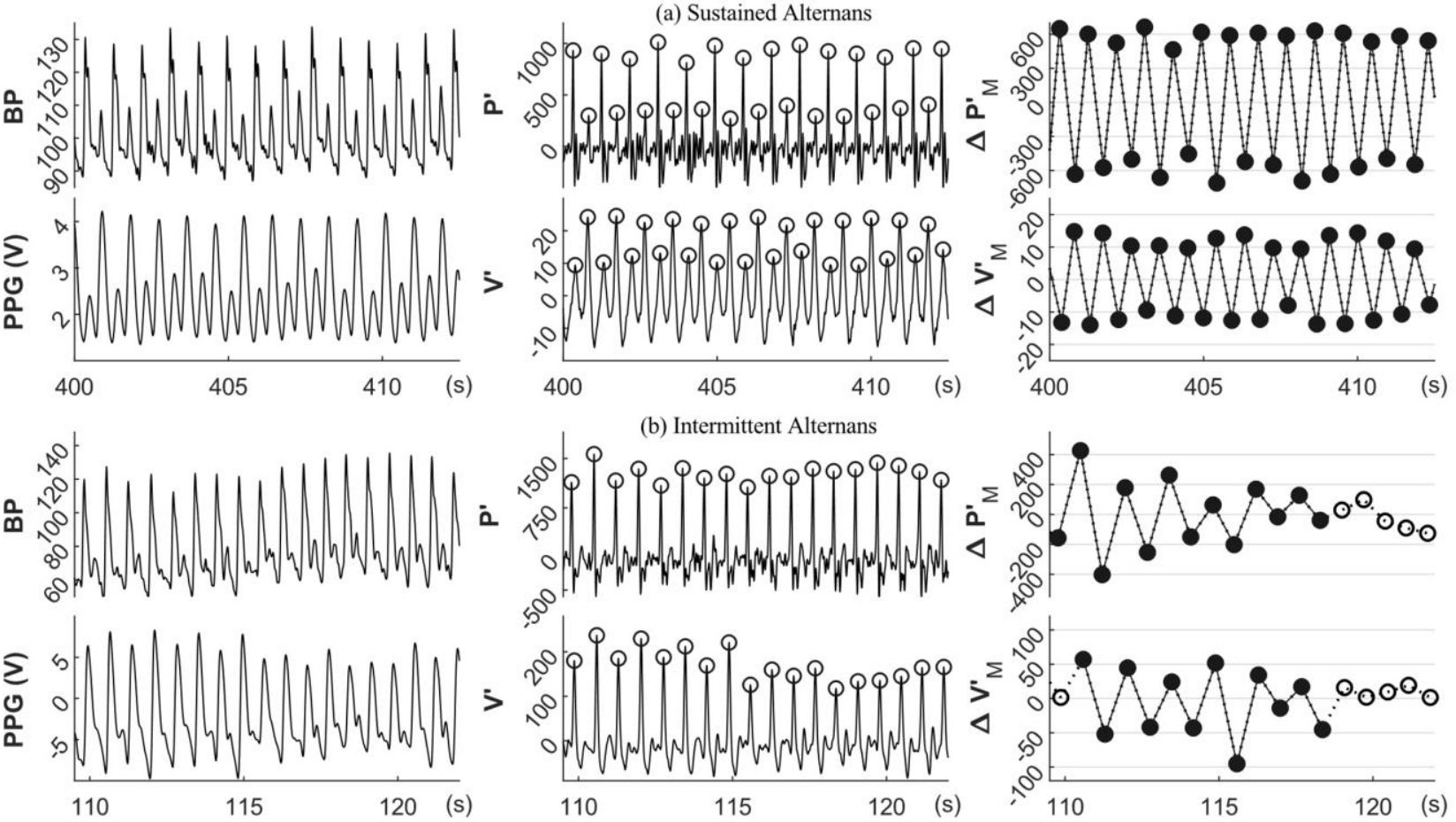

(b) Intermittent Alternans

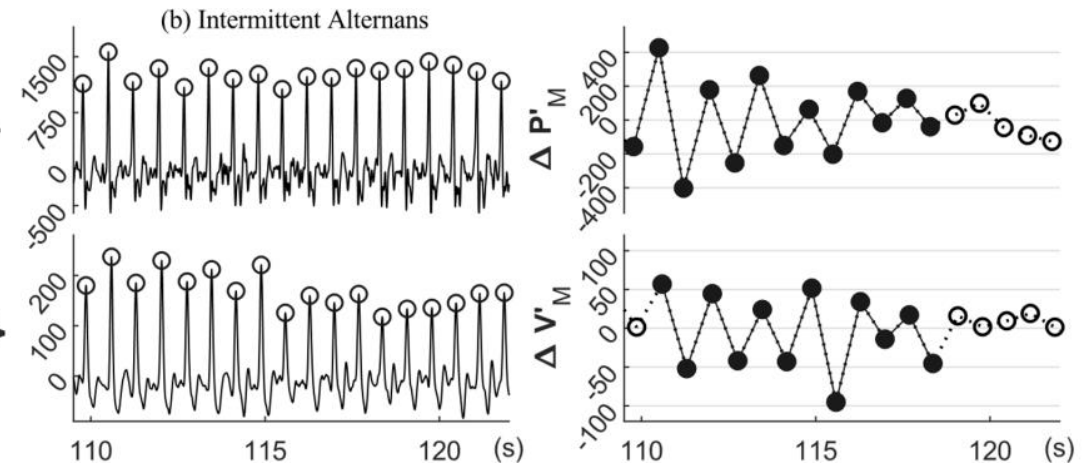

Fig. 2. Recordings showing sustained (a) and intermittent (b) alternans in both BP and PPG. Columns from left to right show the signal (BP in mmHg), its derivative ( $\mathrm{P}^{\prime}$ in $\left.\mathrm{mmHg} / \mathrm{s}\right)$ and the beat to beat changes in the first derivative peaks $\left(\Delta \mathrm{P}^{\prime}{ }_{\mathrm{M}}\right.$ in $\left.\mathrm{mmHg} / \mathrm{s}\right)$. $\mathrm{PPG}(\mathrm{V}), \mathrm{V}$ ' and $\Delta \mathrm{V}^{\prime}{ }_{\mathrm{M}}$ are shown in arbitrary units . In the third column, black circles indicate the duration of mechanical alternans. 

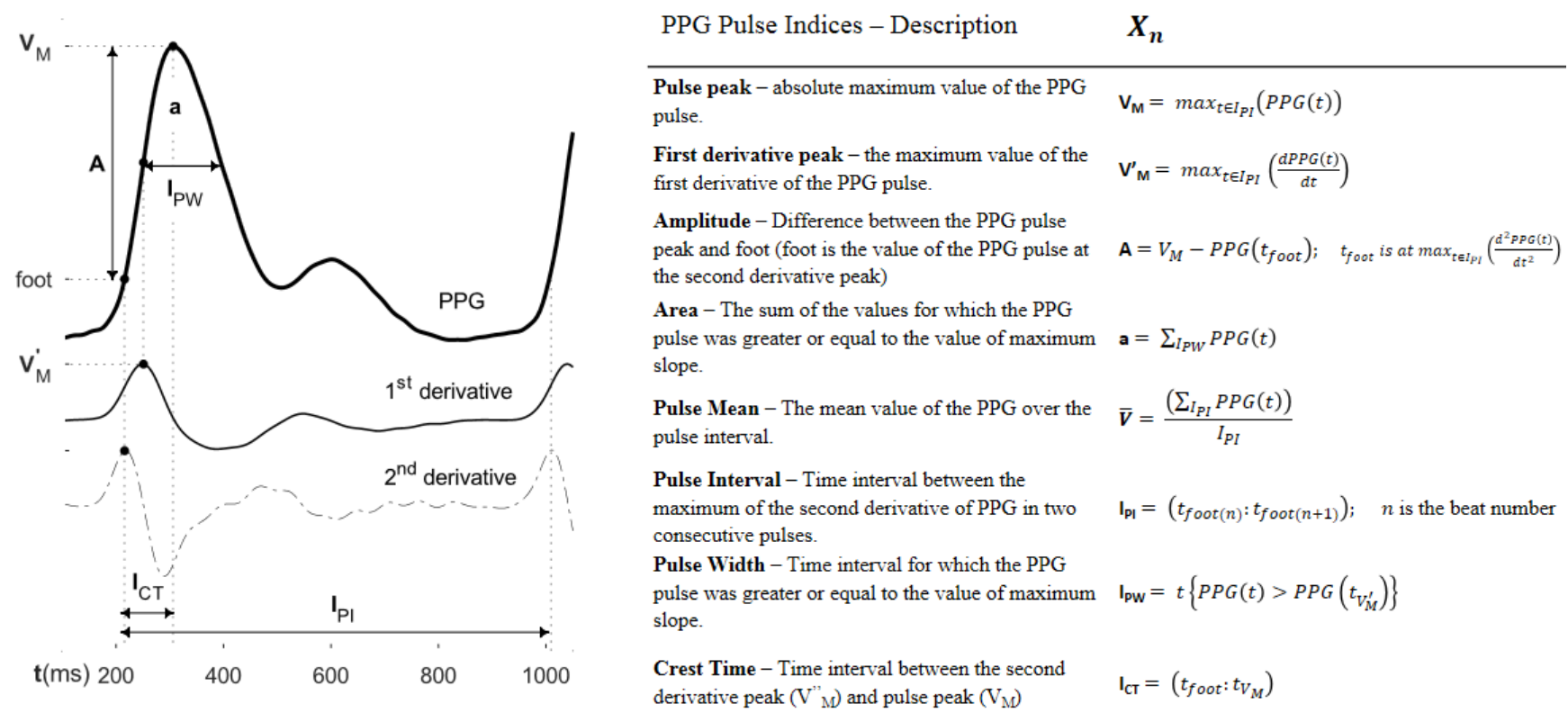

Fig. 3. Definition of PPG indices used in the study. Left: A single PPG pulse. The y-axis represents the magnitude of the signals arbitrary units. Eight pulse specific features are defined and calculated according to the right-hand side equations.

Patients exhibiting at least one MA episode in the PPG were classified as true or false positive, based on the presence of MA in the BP. Similarly, patients that did not exhibit MA in PPG features were classified as true or false negative based on the absence of MA in the BP. Sensitivity and specificity were calculated as the true positive rate and true negative rate, respectively. The range of the best performing threshold values are presented in Table II and III.

\section{RESULTS}

Out of 35 patients, 23 showed intermittent BP MA (13 in Group A and 10 in Group B), 9 of which also exhibited sustained BP MA (all in Group A). The average peak detection accuracy in the PPG recordings was $98.1 \%( \pm 3.93 \%)$; for Group A, 98.1\% ( $\pm 3.37 \%)$, and for Group B, $98.2 \%( \pm 4.63 \%)$.

Tables II and III summarize the performance of PPG-based surrogates for MA, for sustained and intermittent MA respectively. Sensitivity, specificity and accuracy relative to the thresholds identified by ROC analysis are shown along with alternans magnitude correlation.

TABLE III

Intermittent Alternans

\begin{tabular}{|c|c|c|c|c|c|c|c|c|c|c|c|c|c|c|c|}
\hline \multirow{2}{*}{$\begin{array}{c}\text { PPG } \\
\text { Pulse } \\
\text { Indices }\end{array}$} & \multicolumn{5}{|c|}{ Group A } & \multicolumn{5}{|c|}{ Group B } & \multicolumn{5}{|c|}{ Group A+B } \\
\hline & $\begin{array}{c}\text { Threshold } \\
(\%)\end{array}$ & $\begin{array}{l}\mathrm{Se} \\
\%\end{array}$ & $\begin{array}{c}\text { Sp } \\
\%\end{array}$ & $\underset{\%}{\operatorname{Acc}}$ & $\begin{array}{c}\text { MA } \\
\text { Corr }\end{array}$ & $\begin{array}{c}\text { Threshold } \\
(\%)\end{array}$ & $\begin{array}{l}\mathrm{Se} \\
\%\end{array}$ & $\begin{array}{r}\text { Sp } \\
\%\end{array}$ & $\begin{array}{c}\text { Acc } \\
\%\end{array}$ & $\begin{array}{c}\text { MA } \\
\text { Corr }\end{array}$ & $\begin{array}{c}\text { Threshold } \\
(\%)\end{array}$ & $\begin{array}{l}\mathrm{Se} \\
\%\end{array}$ & $\begin{array}{r}\text { Sp } \\
\%\end{array}$ & $\underset{\%}{\operatorname{Acc}}$ & $\begin{array}{l}\text { MA } \\
\text { Corr }\end{array}$ \\
\hline $\mathbf{V}_{M}^{\prime}$ & $(0-20)$ & 100 & 100 & 100 & 0.94 & $(6-8)$ & 100 & 83 & 94 & 0.41 & $(6-8)$ & 100 & 92 & 97 & 0.91 \\
\hline $\mathbf{I}_{\mathbf{P W}}$ & $(0-23)$ & 100 & 67 & 89 & 0.61 & $(0-7)$ & 100 & 67 & 88 & 0.35 & $(0-7)$ & 100 & 67 & 89 & 0.66 \\
\hline $\mathbf{I}_{\mathbf{P I}}$ & $(0-1)$ & 100 & 83 & 95 & 0.90 & $(0-1)$ & 70 & 83 & 75 & 0.08 & $(0-1)$ & 87 & 83 & 86 & 0.81 \\
\hline $\mathbf{A}$ & $(18-21)$ & 100 & 100 & 100 & 0.91 & $(8-10)$ & 80 & 83 & 81 & 0.47 & $(18-21)$ & 78 & 100 & 86 & 0.82 \\
\hline $\mathbf{V}_{\mathbf{M}}$ & $(0-5)$ & 92 & 83 & 89 & 0.20 & $(0-1)$ & 100 & 50 & 81 & 0.76 & $(0-1)$ & 96 & 67 & 86 & 0.15 \\
\hline $\mathbf{a}$ & $(0-40)$ & 100 & 100 & 100 & 0.87 & $(24-26)$ & 70 & 50 & 63 & 0.06 & $(40)$ & 74 & 92 & 80 & 0.70 \\
\hline $\mathbf{I}_{\mathbf{C T}}$ & (8) & 77 & 83 & 79 & 0.73 & $(0-8)$ & 70 & 67 & 69 & 0.07 & (8) & 74 & 75 & 74 & 0.64 \\
\hline$\overline{\mathbf{V}}$ & $(0-3)$ & 85 & 67 & 79 & 0.48 & $(14-15)$ & 60 & 67 & 63 & -0.01 & $(0-3)$ & 78 & 58 & 71 & 0.46 \\
\hline
\end{tabular}

Intermittent MA in BP was present in 13 patients in group A and 10 patients in group B. 

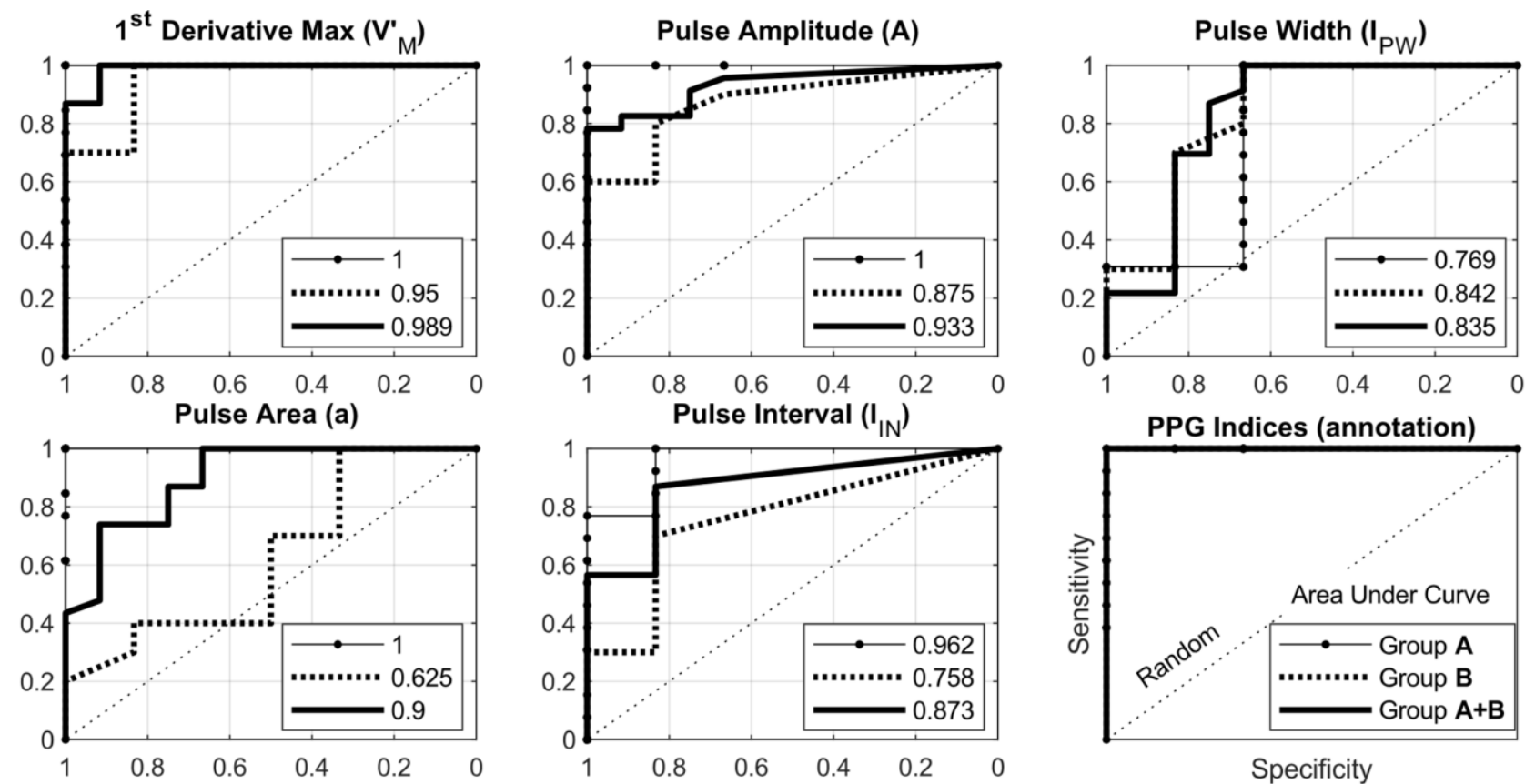

Fig. 4. Mechanical Alternans categorization performance for the top 5 PPG indices. There are 23 patients with intermittent alternans in BP and 12 without. ROC curves are constructed by varying the alternans relative magnitude threshold between 0 and $60 \%$.

ROC curves for the top 5 performing indices are shown in Fig. 4. Based on the area under the curve, $V^{\prime}{ }_{M}$, was the best surrogate for MA detection, providing $100 \%$ sensitivity and $100 \%$ specificity in Group A for both sustained and intermittent alternans, and $100 \%$ sensitivity and $83 \%$ specificity in Group B for intermittent MA. For intermittent MA defined by different thresholds and window lengths, the results are similar. They are presented in supplementary Table S. I.

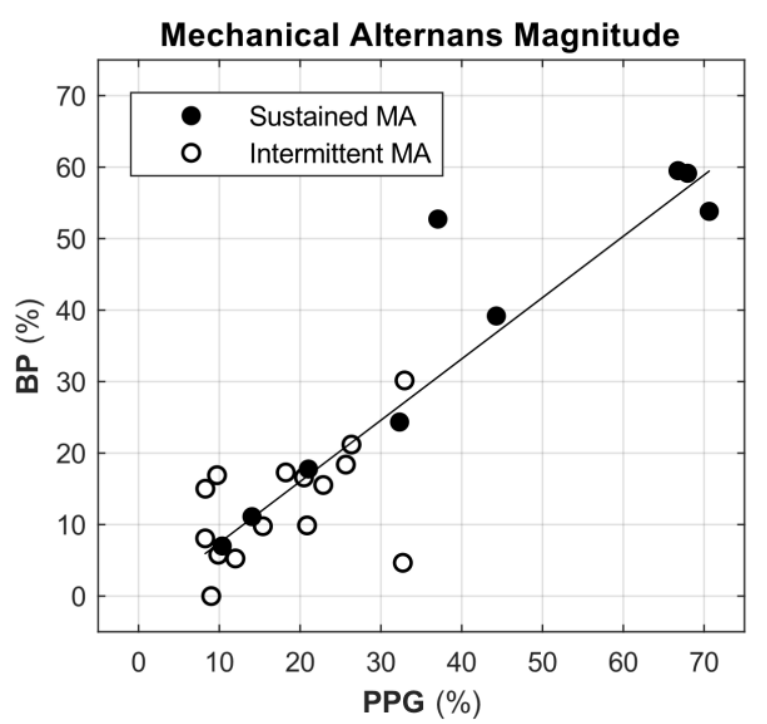

Fig. 5. Magnitude correlation for MA in BP and PPG $V_{M}^{\prime}$. Each point represents a patient with either sustained or intermittent alternans. The coordinates represent the mean relative magnitude of all the alternans episodes detected in a patient. The line fitting was done for all the patients, $\mathrm{R}=0.904$. For patients with intermittent and sustained MA, the correlations were $\mathrm{R}=0.51$ and $\mathrm{R}=0.95$ respectively.

Indices based on pulse morphology, such as pulse amplitude
(A and $\mathbf{V}_{\mathbf{M}}$ ) and area (a) also performed well, with accuracy $>85 \%$ for both sustained and intermittent (group A+B) alternans. Interestingly, pulse interval also provided good accuracy in the detection of MA, possibly as it is affected by ventricular contractility. Patients with sustained alternans were detected with high accuracy ( $>89 \%$ ) by all the PPG indices. Intermittent alternans was detected with greater accuracy in Group A than in Group B.

The correlation between the magnitude of MA and the magnitude of PPG-based MA was high, and greater in Group A than B (Tables II and III).

Index $V^{\prime}{ }_{M}$ outperformed the other indices, showing a Pearson correlation equal to $0.94,0.41$ and 0.91 for Groups $\mathrm{A}, \mathrm{B}$ and $\mathrm{A}+\mathrm{B}$ respectively. A scatter plot of the relative magnitudes of alternans in $V^{\prime}{ }_{M}$ in each patient is presented in Fig. 5. Patients that exhibited alternans only in the PPG were included in the correlation analysis and were considered to have zero magnitude of alternans in BP.

Among the 23 alternans positive patients, 258 episodes of MA (both intermittent and sustained) were detected in the blood pressure and 273 episodes were detected in the PPG.

In Group B, where heart rate varied due to exercise it was possible to study the interaction between occurrence of MA and heart rate. Here, there were 25 episodes of (intermittent) MA in $\mathrm{BP}$ and 26 episodes of MA in the PPG $\left(V^{\prime}{ }_{M}\right)$.

The magnitude spread of these episodes and the occurrence within normalized heart rate intervals is illustrated in Fig. 6. Normalization was done with respect to the predicted maximum heart rate of each patient. The results are very similar between $\mathrm{BP}$ and $V{ }^{\prime}{ }_{M}$. The relative magnitude of alternans was higher for $V^{\prime}{ }_{M}$ closer to the maximum predicted heart rate, however the frequency of episodes within the heart rate intervals was akin to episodes of MA in BP. 


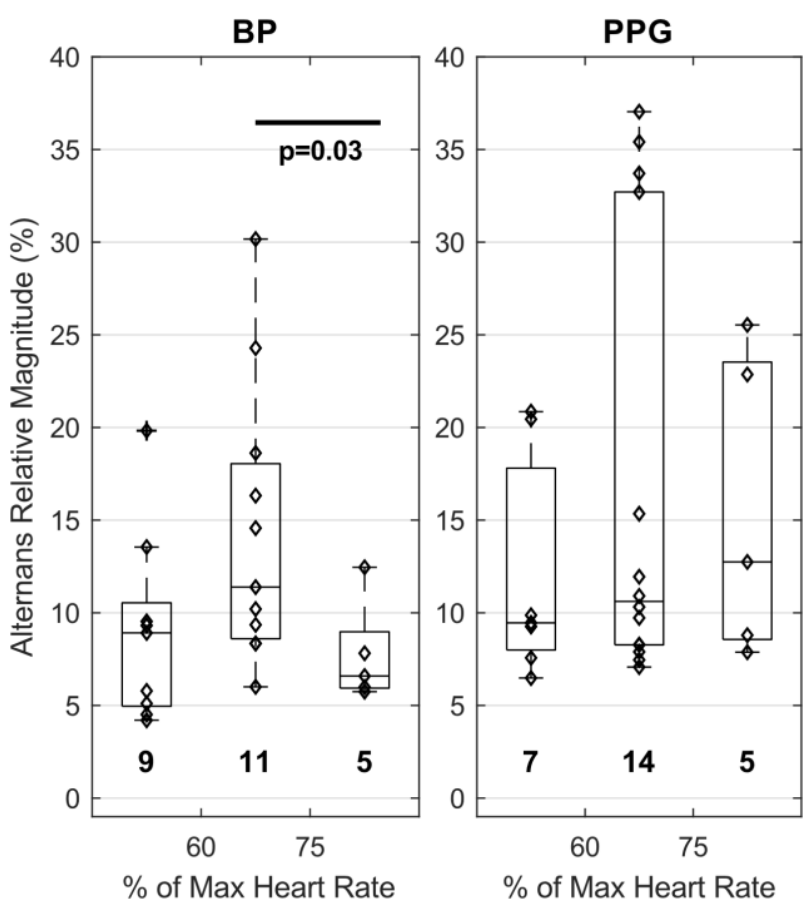

Fig. 6. Interactions between heart rate and magnitude of alternans episodes in BP and PPG $V^{\prime}{ }_{M}$. The number of alternans events within each heart rate interval is presented under each group. Statistically significant differences were calculated using the Mann-Whitney U ranked test.

\section{DISCUSSION}

This study demonstrates for the first time that patients with MA can be accurately identified using the PPG signals alone and may have important implications in facilitating risk stratification in heart failure. Although PPG oscillations have been previously reported anecdotally during MA [27], [28], to the extent of our knowledge, this is the first quantitative investigation of PPG-based surrogates for MA.

Two groups of patients were investigated: Group A, where BP was measured invasively and Group B where it was measured non-invasively. Furthermore, in Group A, MA was induced either by pacing or by sustained stable VT, whereas in Group B, MA was induced by exercise, through CPET. Sustained alternans was solely present in Group A, possibly because patients undergoing catheter ablation for ventricular tachycardia or cardiac surgery for coronary artery bypass grafting had poorer ventricular function than patients in group $\mathrm{B}$ and because ventricular pacing/tachycardia is known to facilitate the establishment of MA [6]. Detection of MA was more challenging in Group B than in Group A not only because exercise less effective than ventricular pacing/tachycardia in inducing MA and patients had better ventricular function, but also because the reference signal for MA in this group, i.e. blood pressure, was measured non-invasively and measurements were more prone to movement artefacts due to exercise. As expected, all PPG indices performed better in Group A than in Group B.

Alternans was categorized as sustained or intermittent. Sustained alternans has been more widely investigated [2]-[8], [12] and its prognostic value has been demonstrated in several studies [3], [6], [8]. We also considered intermittent MA, which occurs sporadically in short episodes and often shows lower magnitude, because we hypothesized that this may be useful as an earlier predictor of adverse cardiovascular outcome. Further investigation is needed to assess the prognostic value of both sustained and intermittent PPG-derived MA.

The PPG first derivative pulse peak, $V_{M}{ }_{M}$, was the best surrogate for MA, with $100 \%$ sensitivity and $92 \%$ specificity for intermittent MA in group $\mathrm{A}+\mathrm{B}$. In Group $\mathrm{A}$, both sustained and intermittent MA were detected with $100 \%$ sensitivity and $100 \%$ specificity using both the maximum of the pulse derivative $\left(V^{\prime}{ }_{M}\right)$ and pulse amplitude $(A)$.

The magnitude of MA was highly correlated between BP and PPG indices $V^{\prime}{ }_{M}$, pulse interval and pulse amplitude (Tables IIIIV). The correlation was higher for patients with sustained alternans (Fig. 5). The lower correlation of intermittent alternans can be explained by the low MA magnitude values ( $<35 \%$ ) for both BP and PPG. In Group B, the correlation was also lower for most indices, possibly on account of movement artifacts related to exercise and apparatus limitations in detecting BP non-invasively [29].

In Table III we presented the best performing threshold values for each group individually, as well as for group A and B combined. In Group A, accuracy can be maximized using a wide range of thresholds, while in Group B accuracy is maximized using similar thresholds but within a narrower window, since the magnitude of mechanical alternans was generally lower in this group.

The PPG pulse interval index was a good surrogate for MA, detecting sustained and intermittent MA with $95 \%$ and $86 \%$ accuracy, respectively. Interesting, small pulse interval variations allowed to accurately detect MA even when the heart rate was fixed (Group A). Similar variations have been reported before in pulse arrival time during pacing [30]. Since pulsations of higher pressure travel faster through arteries [31], we hypothesize that this may be due to beat-to-beat variations in contractility directly translating into variations in the pulse arrival time and therefore in the pulse interval.

Fig. 6 shows a qualitative trend of MA magnitude dependence on heart rate - very similar to what is present in [7], where MA generated through atrial pacing also shows a heart rate dependent peak magnitude. We believe this is an area that needs to be further examined, as literature provides little information on this.

Our hypothesis was that PPG could be used to track oscillations in the blood pressure, and therefore MA could be detected via PPG regardless of the underlying mechanisms, due to the intrinsic link between blood pressure and arterial flow [32]. Previous studies have shown that MA can be observed in both ventricular blood pressure [12] and in the aortic ejection, via Doppler measurements [33]. MA has also been detected in the peripheral arterial blood pressure [8], with significant increase in magnitude compared to left ventricle alternans. Due to peripheral blood pressure augmentation [34] and the PPG's ability to detect changes in peripheral blood flow [35], it was assumed that alternans could be optically detected at peripheral sites, i.e. finger, wrist. 
While this study demonstrates that the PPG is a technology that can track beat to beat blood pressure oscillations, its use for accurate cuffless blood pressure estimation remains a challenging task [24]-[26].

\section{A. Definition of Mechanical Alternans}

The definitions of MA have varied slightly in literature, however there seems to be a consensus that MA indicates poorer prognosis when it is sustained and has higher magnitude. Sustained alternans has often been defined as a systolic or $\mathrm{dP} / \mathrm{dt}$ alteration over 20 beats or more. A threshold is commonly employed to discard low magnitude alternans, i.e. $4 \mathrm{mmHg}$ [3] for systolic BP, $100 \mathrm{mmHg} / \mathrm{s} \mathrm{[7]} \mathrm{for} \mathrm{max} \mathrm{dP} / \mathrm{dt}$, particularly in clinical studies. However, there are also studies looking at microsystolic blood pressure alternans (MBPA) [8] which consider alternans of all magnitudes.

In this study, the definition of sustained alternans was maintained as 20 alternating beats or more, and an adaptive threshold was introduced, $4 \%$ relative change to the stronger beat. In our study population, a $4 \%$ change in peak $\mathrm{dP} / \mathrm{dt}, P^{\prime}{ }_{M}$, corresponds to $33( \pm 24 \mathrm{mmHg} / \mathrm{s})$.

Intermittent alternans were defined as 12 consecutive beats or more to avoid evaluating unwanted short variations. It has been shown that similar variations [36], which we wanted to exclude from our analysis, can occur from ectopic beats and last for up to 8-10 beats. 12 was chosen to allow us to evaluate the shortest MA episodes while also excluding unwanted variations.

Mechanical alternans could also be defined in terms of a spectral power index, if the spectral method is used for detection. However, the spectral method is limited in assessing intermittent alternans, and its sensitivity is susceptible to ectopics and noise [37].

\section{B. Clinical Perspective}

PPG-derived MA may represent a novel useful prognostic marker in patients with heart failure. The ability to detect MA with existing, widely available medical equipment, opens avenues for further clinical investigations into its prognostic utility to risk stratify patients who may benefit from an implantable cardioverter defibrillator or ventricular assist device. With the expansion of wearable devices and m-health, one could speculate that MA could be integrated into an adaptive multi-variate risk-prediction model based on data recorded through a PPG sensor continuously monitoring heart rate, heart rate variability [14], turbulence [21] and respiratory rate [15], [16]. The combination of predictors related to complementary pathophysiological mechanisms provides better risk profiling [38] and an opportunity that should be tested in future studies.

\section{Limitations and Future Studies}

Studies with longer recording times are needed to assess the accuracy during long term monitoring. In our cohort the average recording time was $16 \mathrm{~m} 26 \mathrm{~s}( \pm 12 \mathrm{~m} 15 \mathrm{~s})$ and it was limited by the procedure or patient fitness.

The continuity required for our analysis was affected, in Group B, by frequent recalibrations of the Finometer.
Movement artifacts affect both the PPG and noninvasive BP. Although noise reduction in the PPG is an active field of study, sophisticated techniques of signal reconstruction during movement have focused on accurate estimations of heart rate [39], [40]. PPG motion artifact reduction remains a challenging task. In this study, PPG has been recorded in stable, and mild movement conditions, as per clinical procedure.

The presence of alternans was investigated independently in each PPG pulse feature, in order to find a direct surrogate for MA that is easy to measure and implement. Although the results in our cohort have been satisfactory for several PPG features, a multi-variable analysis and machine learning techniques could be used to further improve the ability of PPG to detect alternans. These are more likely to be needed in challenging recording settings, such as wearable wrist devices, where movement artifacts are frequent and skin perfusion is low. This optimization could enable more widespread use of PPG data both in the hospital setting and for home monitoring to enable treatment and risk stratification in community care.

\section{CONCLUSION}

This study demonstrates that blood pressure mechanical alternans, an independent marker of cardiovascular impairment and predictor of poor outcome in heart failure patients, can be detected using PPG-based surrogates. This novel methodology opens new possibilities for improving risk stratification of cardiac patients, but future studies are needed to determine its clinical implications and impact.

\section{REFERENCES}

[1] L. Traube, "Ein Fall von Pulsus Bigeminus nebst Bemerkungen uber die Leberschwellungen bei Klappenfehlern and uber acute Leberatrophie," Berlin Klin Wochenschr, vol. 9, pp. 185-188, 1872. D. E. Euler, "Cardiac alternans-mechanisms and pathophysiological significance," Cardiovasc Res, vol. 42, pp. 58390, 1999.

[3] R. Kim et al., "Mechanical alternans is associated with mortality in acute hospitalized heart failure: prospective mechanical alternans study (MAS)," Circ. Arrhythmia Electrophysiol., vol. 7, no. 2, pp. 259-266, Apr. 2014.

[4] P. D. White, "Alternation of the pulse: a common clinical condition," American Journal of Medical Sciences, vol. 150. pp. 8297, 1915.

[5] M. Kodama et al., "Mechanical alternans in patients with chronic heart failure.," J. Card. Fail., vol. 7, no. 2, pp. 138-145, 2001.

[6] A. Hirashiki et al., "Prognostic value of pacing-induced mechanical alternans in patients with mild-to-moderate idiopathic dilated cardiomyopathy in sinus rhythm," J. Am. Coll. Cardiol., vol. 47, no. 7, pp. 1382-1389, Apr. 2006.

[7] T. Kashimura et al., "Mechanical alternans in human idiopathic dilated cardiomyopathy is caused with impaired force-frequency relationship and enhanced poststimulation potentiation," Heart Vessels, vol. 28, no. 3, pp. 336-344, 2013.

[8] R. J. Selvaraj, A. Suszko, A. Subramanian, S. Mak, R. Wainstein, and V. S. Chauhan, "Microscopic systolic pressure alternans in human cardiomyopathy: Noninvasive evaluation of a novel risk marker and correlation with microvolt T-wave alternans," Hear. Rhythm, vol. 8, no. 2, pp. 236-243, Feb. 2011.

[9] W. Parmley, W, H. Tomoda, S. Fujimura, and M. Matloff, Jack, "Relation between pulsus alternans and transient occlusion of the left anterior descending coronary artery," Cardiovasc. Res., vol. 6 , pp. 709-715, 1972.

[10] K. E. Cohn, H. Sandler, and E. W. Hancock, "Mechanisms of pulsus 
alternans.," Circulation, vol. 36, no. 3, pp. 372-80, 1967.

[11] O. M. Hess, E. P. Surber, M. Ritter, and H. P. Krayenbuehl, "Pulsus alternans: Its influence on systolic and diastolic function in aortic valve disease," J. Am. Coll. Cardiol., vol. 4, no. 1, pp. 1-7, 1984.

[12] B. Surawicz and C. Fisch, "Cardiac alternans: Diverse mechanisms and clinical manifestations," J. Am. Coll. Cardiol., vol. 20, no. 2, pp. 483-499, 1992.

[13] C. McCarthy, N. Pradhan, C. Redpath, and A. Adler, "Validation of the Empatica E4 wristband," 2016 IEEE EMBS Int. Student Conf. Expand. Boundaries Biomed. Eng. Heal. ISC 2016 - Proc., pp. 4-7, 2016.

[14] E. Gil, M. Orini, R. Bailón, J. M. Vergara, L. Mainardi, and P. Laguna, "Photoplethysmography pulse rate variability as a surrogate measurement of heart rate variability during non-stationary conditions," Physiol. Meas., vol. 31, no. 9, pp. 1271-1290, 2010.

[15] M. Orini, M. D. Pelaez-Coca, R. Bailon, and E. Gil, "Estimation of spontaneous respiratory rate from photoplethysmography by cross time-frequency analysis," Proc. Comput. Cardiol., vol. 2, no. i, pp. 661-664, 2011.

[16] M. D. Pelaez-Coca, M. Orini, J. Lazaro, R. Bailon, and E. Gil, "Cross time-frequency analysis for combining information of several sources: Application to estimation of spontaneous respiratory rate from photoplethysmography," Comput. Math. Methods Med., vol. 2013, p. 631978, 2013.

[17] J. Lázaro, E. Gil, J. M. Vergara, and P. Laguna, "Pulse rate variability analysis for discrimination of sleep-apnea-related decreases in the amplitude fluctuations of pulse

photoplethysmographic signal in children," IEEE J. Biomed. Heal. Informatics, vol. 18, no. 1, pp. 240-246, 2014.

[18] E. Gil, M. Mendez, J. M. Vergara, S. Cerutti, A. M. Bianchi, and P. Laguna, "Discrimination of sleep-apnea-related decreases in the amplitude fluctuations of ppg signal in children by HRV analysis," IEEE Trans. Biomed. Eng., vol. 56, no. 4, pp. 1005-1014, 2009.

[19] P. Dehkordi et al., "Evaluation of cardiac modulation in children in response to apnea/hypopnea using the Phone Oximeter ${ }^{\mathrm{TM}}$," Physiol. Meas., vol. 37, no. 2, pp. 187-202, 2016.

[20] A. Sološenko, A. Petrènas, and V. Marozas, "Photoplethysmography-based method for automatic detection of premature ventricular contractions," vol. 9, no. 5, pp. 662-669, 2015.

[21] E. Gil, L. Sornmo, and P. Laguna, "Detection of heart rate turbulence in photoplethysmographic signals," 2011 Comput. Cardiol., pp. 665-668, 2011.

[22] V. D. A. Corino, R. Laureanti, L. Ferranti, G. Scarpini, F. Lombardi, and L. T. Mainardi, "Detection of atrial fibrillation episodes using a wristband device," Physiol. Meas., vol. 38, no. 5, pp. 787-799, 2017.

[23] S.-C. Tang et al., "Identification of atrial fibrillation by quantitative analyses of fingertip photoplethysmogram," Sci. Rep., vol. 7, no. March, p. 45644, 2017.

[24] R. Mukkamala et al., "Toward ubiquitous blood pressure monitoring via pulse transit time: Theory and practice," IEEE Trans. Biomed. Eng., vol. 62, no. 8, pp. 1879-1901, 2015.

[25] X. Ding, B. P. Yan, Y.-T. Zhang, J. Liu, N. Zhao, and H. K. Tsang, "Pulse transit time based continuous cuffless blood pressure estimation: A new extension and a comprehensive evaluation," Sci. Rep., vol. 7, no. 1, p. 11554, 2017.

[26] J. Bolea et al., "Pulse rate and transit time analysis to predict hypotension events after spinal anesthesia during programmed cesarean labor," Ann. Biomed. Eng., vol. 45, no. 9, pp. 2253-2263, 2017.

[27] M. Saghaei and M. Mortazavian, "Pulsus alternans during general anesthesia with halothane," vol. 93, no. 1, pp. 91-94, 2000.

[28] M. Weber, "Pulsus alternans. A case study.," Crit. Care Nurse, vol. 23, no. 3, pp. 51-54, 2003.

[29] L. W. J. Bogert and J. J. van Lieshout, "Non-invasive pulsatile arterial pressure and stroke volume changes from the human finger," Exp. Physiol., vol. 90, no. 4, pp. 437-446, 2005.

[30] S. van Duijvenboden, N. Child, B. Hanson, J. Gill, P. Taggart, and M. Orini, "Pulse arrival time accurately detects pacing-induced mechanical alternans," Comput. Cardiol. (2010)., vol. 44, pp. 2-5, 2017.

[31] J. C. Bramwell and A. V. Hill, "The velocity of the pulse wave in man,” Proc. R. Soc. B Biol. Sci., vol. 93, no. 652, pp. 298-306, 1922.
[32] W. W. Nichols, M. F. O'Rourke, C. Vlachopolus, and D. A. McDonald, McDonald's Blood flow in arteries: theoretical, experimental and clinical principles, 6th ed. London: London : Hodder Arnold, 2011.

[33] A. Garcia-Lledo, M. A. San Martin, E. Diaz-Caraballo, and J. Balaguera, "Mechanical alternans detected by doppler-derived dP/dt," Rev. Esp. Cardiol., vol. 63, no. 9, pp. 1100-9, 2010.

[34] M. Karamanoglu, M. O'ROURKE, A. Avolio, and R. Kelly, “An analysis of the relationship between central aortic and peripheral upper limb pressure waves in man," Eur. Heart J., no. 1993, pp. 160-167, 1993.

[35] J. Allen, "Photoplethysmography and its application in clinical physiological measurement," Physiol. Meas., vol. 28, no. 3, pp. R1R39, 2007.

[36] B. Friedman, "Alternation of cycle length in pulsus alternans," Am. Heart J., vol. 51, no. 5, pp. 701-712, 1956.

[37] M. Orini et al., "Comparative evaluation of methodologies for Twave alternans mapping in electrograms," IEEE Trans. Biomed. Eng., vol. 61, no. 2, pp. 308-316, 2014.

[38] J. Ramírez et al., "Sudden cardiac death and pump failure death prediction in chronic heart failure by combining ECG and clinical markers in an integrated risk model," PLoS One, vol. 12, no. 10, pp. $1-15,2017$.

[39] D. Dao et al., "A robust motion artifact detection algorithm for accurate detection of heart rates from photoplethysmographic signals using time-frequency spectral features," IEEE J. Biomed. Heal. Informatics, vol. 21, no. 5, pp. 1242-1253, 2017.

[40] Y. Ye, Y. Cheng, W. He, M. Hou, and Z. Zhang, "Combining nonlinear adaptive filtering and signal decomposition for motion artifact removal in wearable photoplethysmography," IEEE Sens. J., vol. 16, no. 19, pp. 7133-7141, 2016. 